\title{
Gearbox Noise and Vibration Prediction and Control
}

\author{
Jiri Tuma \\ VSB-Technical University of Ostrava, Ostrava, Czech Republic
}

(Received 30 April 2009; accepted 14 May 2009)

This paper will review practical techniques and procedures employed to quiet gearboxes and transmission units. The author prefers solving the gear noise problem at the very source to introduce an enclosure as a means to reduce radiated noise, which seems to be easy but its effect on the sound pressure level is small. The gearbox noise problem solution is focused on the improvement of gear design; on the verification of its effect on the radiated noise and the determination of the gears' contribution to the truck's or car's overall noise levels and on the analytical and/or numerical computer-based tools needed to perform the signal processing and diagnostics of geared axis systems. All of the analytical methods are based on the time and frequency domain approach. Special care is addressed to the smoothness of the drive resulting from the transmission error variation during a mesh cycle. This paper will review the progress in technique of the gear angular vibration analysis and its effect on gear noise due to the self excited vibration. This presentation will include some examples of the use of such approaches in practical engineering problems.

\section{INTRODUCTION}

The major functions of a gearbox are to transform speed and torque in a given ratio and to change the axis of rotation. The main parts of the gearbox are the gears, shafts, bearings, housing, and the outside of the gearbox clutches and couplings. The gears are machine elements that transmit motion by means of successively engaging teeth. The transformation of speed is without slip and loss of synchronization. Thus, the relationship between the angular velocities of the driving gear to the driven gear, or the velocity ratio, of a pair of mating teeth results from the number of teeth of the driving and driven gear. A gear train consists of two or more gears (a pinion and wheels), which serve to transmit motion from one axis to another. The gear train operational condition depends on the drive systems. Some gearboxes work with the steady-state rotational speed while the rotational speed of the others varies in a certain range. Independently on the rotational speed, the transmitted power can be constant or variable.

The gearbox is a source of vibration and, consequently, noise. Except for bearing fatal defects or extreme structureresonance amplification, gears are the main sources of high frequency vibration and noise, even in newly built units. The gearbox's overall sound pressure level (SPL), compared to the SPL associated with the meshing gears, is only by some $5 \mathrm{~dB}$ higher at maximum. There are two possible solutions for keeping a transmission unit quiet. Introducing an enclosure to prevent noise radiation - with the consequences of low efficiency and difficult maintenance - is the easiest one. The more sophisticated and much more efficient solution is based on solving the noise problem at the very source. It means to introduce improvement aimed at the gear design and manufacturing, which results in the greatest reduction of the SPL.

\section{HISTORICAL NOTES}

The author of this paper was a member of the team for gearbox noise reduction at TATRA, a Czech company that pro- duces off-road and on-road trucks. TATRA trucks are used to carry goods and have a maximum authorized total mass exceeding 12 tonnes. In the past, TATRA truck gearboxes were noted for a very robust design. Their only disadvantage was that they were "a little noisy." The introduction of the $80 \mathrm{~dB}$ limit for the peak pass-by noise level of on-road trucks in Europe in 1994 created a challenge to start research works on gearbox noise reduction. It should be noted that the pass-by noise measurements shall be made using the time Fweighting and the fre-quency A-weighting on the $\mathrm{dB}$ scale at a distance of $7.5 \mathrm{~m}$ from the centre of the test track. In the USA, the SPL is measured at the distance of 50 feet (doubled in comparison to the European test method), therefore $6 \mathrm{~dB}$ has to be added to the USA limit to make it correspond to the European level. The truck's operational condition during pass-by noise measurements is based on the full accel-eration test specified in the International Standard, ISO R362-1982 (E) "Acoustic-Measurement of Noise Emitted by Accelerating Road Vehicles-Engineering Methods" or SAE J366 Surface Vehicle standard - (R) "Exterior Sound Level for Heavy Trucks and Buses (Issued 1969-07, Reaffirmed 1987-02).” The test requires the vehicle to be driven through a test track at full acceleration.

The noise level of road vehicles was decreased in intervals as it was possible to fulfil the requirements given by the vehicle noise legislation. The peak SPL of the mentioned category of trucks was set to $84 \mathrm{~dB}$ before 1994 . The $84 \mathrm{~dB}$ trucks were easy to produce but the last decrease of the SPL by $4 \mathrm{~dB}$ required fundamental improvements. The truck engine is the noisiest unit. Engine noise reduction for TATRA trucks of the T 815-2 type, introduced in production in 1989, was reached by using covers and shields that fit over the engine compartment and, also, by reducing the rate of the rise in cylinder pressure. the reduction of the SPL to reach the last $4 \mathrm{~dB}$ was not easy. To solve the gearbox noise problem it was necessary to decide if the special enclosure should be extended over the gear-box or not. As mentioned before, the designers decided to 